\title{
Increased IL-6 and IL-4 in exhaled breath condensate of patients with nasal polyposis
}

\author{
G.E. Carpagnano1, P. Carratù2, M. Gelardi3, A. Spanevello4, G. Di Gioia², \\ T. Condreva 3 , O. Resta2 ${ }^{2}$ M.P. Foschino Barbaro ${ }^{1}$
}

\begin{abstract}
Increased IL-6 and IL-4 in exhaled breath condensate of patients with nasal polyposis. G.E. Carpagnano, P. Carratù, M. Gelardi, A. Spanevello, G. Di Gioia, T. Condreva, O. Resta, M.P. Foschino Barbaro.

Background and Aim. Nasal polyposis (NP) occurs in about $1-4 \%$ of the worldwide population. Increased plasma concentrations of different pro-inflammatory cytokines have been observed in NP, and might be related to the pathogenesis of this syndrome.The present study was designed to investigate IL-6 and IL-4 concentrations in nasal and oral exhaled breath condensate of patients with early and advanced NP, and following polypectomy.

Methods. Ten individuals with polyposis in early status, twenty-three patients affected by advanced status of
\end{abstract}

\begin{abstract}
NP and ten healthy controls were enrolled into the study. Exhaled breath condensate was collected by all individuals, according to a previous standardised method. An immunoassay kit was used to measure IL-6 and IL-4 levels.

Results. Concentrations of oral and nasal exhaled IL6 and IL-4 were significantly higher in patients with early nasal polyposis and advanced nasal polyposis, compared to healthy controls. A statistically significant decrease of nasally but not of orally exhaled IL-6 $(p<0.001)$ and IL-4 $(p<0.05)$ was observed after polypectomy.

Conclusions. We consider oral and nasal exhaled condensate of IL-6 and IL-4 as valid inflammatory and oxidative stress marker in patients with nasal polyposis. Monaldi Arch Chest Dis 2009; 71: 1, 3-7.
\end{abstract}

Keywords: Exhaled breath condensate, Nasal polyposis, IL-6, IL-4, Polypectomy.

I Section of Respiratory Diseases, Department of Occupational and Medical Sciences, Faculty of Medicine, University of Foggia;

2 Institute of Respiratory Disease, University of Bari;

3 Institute of Otorhinolaryngology, University of Bari;

4 Fondazione Salvatore Maugeri, IRCCS, Cassano Murge, Italy.

Correspondence: Giovanna Elisiana Carpagnano,Via De Nicolò 5, 70121 Bari, Italy; e-mail: ge.carpagnano@unifg.it

\section{Introduction}

Nasal polyposis (NP) is a multifactorial disease involving nasal mucosa, and occuring in about $1-4 \%$ of the population [1]. Increased plasma concentrations of different Th1 (TNF-alpha, IL-1, IL-6), Th2 (IL-5, IL-4) cytokines, and other inflammation-related mediators have been found in NP [2]. Eosinophils are the most abundant cell type, indeed high IgE concentrations have been found in NP [3]. Several pro-infiammatory cytokines, such as IL-4 and IL-5 have been implicated in inducing eosinophils production, eliciting tissue damage and finally promoting airway remodelling in NP [4]. It has been recently proposed that nasal polyposis can propagate from upper to lower airways [5]; however, mechanisms binding upper and lower airway inflammation in NP remain poorly understood. Two theories are mainly accepted and include an activation of nasal reflexes, or pulmonary aspiration of nasal contents [6].

Several methods are available to collect and analyse samples from upper and lower airways, such as bronchial and nasal biopsy, bronchoalveolar lavage (BAL), nasal lavage, nasal brushing, and nasal secrete collection. They are invasive or semiinvasive assays, need specific competence, and are non-repeatable in clinical practice. These impediments enhance the importance of assessing an easy, alternative, and non-invasive method to better ex- plore the upper and lower respiratory system and to acquire additional features in nasal polyposis.

The exhaled breath condensate is a new, noninvasive method, is able to detect inflammatory and metabolic markers of the airways and can be performed with good reproducibility [7]. Recently, Griese and colleagues modified breath condensate collections by applying a nasal prong to the device, usually used for oxygen delivery [8]. This new adapted method is more comfortable and efficient to assemble the exhaled air condensate from the upper airways (including nasal cavities) [9]. Several inflammatory markers such as IL-1ß [10], IL8 [10], IL-6 [11], IL-10 [12], TNF- $\alpha$ [10] and $\mathrm{LTB}_{4}[13]$ have been observed in orally exhaled breath condensate of individuals affected by different respiratory diseases. The present study was designed to investigate IL- 6 and IL-4 concentrations in nasally and orally exhaled breath condensate of healthy subjects and of patients with early (grade 1) and advanced (grade 2-3) NP, to verify whether these markers are increased in patients with NP and change after polypectomy.

Material and Methods

\section{Study design}

We enrolled into the study ten patients with polyposis in early status $\left(41 \pm 9\right.$ years, $\mathrm{FEV}_{1}$ 
95.1 \pm 1.2 , FVC $93.6 \pm 2.2 \%$, BMI $24 \pm 0.4 \mathrm{~kg} / \mathrm{m}^{2}$ ), twenty-three patients affected by advanced status of NP $\left(46 \pm 3\right.$ years, $\mathrm{FEV}_{1} 105.5 \pm 2.1$, FVC $98.4 \pm 2.3 \%$, BMI $\left.25 \pm 0.6 \mathrm{~kg} / \mathrm{m}^{2}\right)$, and ten healthy

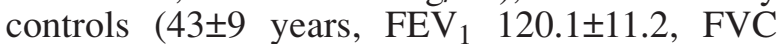
$119 \pm 3.4 \%$, BMI $\left.24 \pm 0.5 \mathrm{~kg} / \mathrm{m}^{2}\right)$. Both, patients and healthy controls were Caucasian and selected from the Department of Otorhinolaryngology, University of Bari. Informed written consent was obtained by all subjects. The study was approved by the Institutional Ethics Committee. The diagnosis of NP was made endoscopically and classified according to the International Conference on Sinus Disease [14]. All patients selected into the study were nonsmokers, did not have nasal, bronchial, or lower respiratory tract infection, as well as chronic cough before the enrollment and/or during the study period. We excluded from the study patients affected by bronchial asthma [15] and individuals with bronchial hyperresponsiveness, according to methacholine provocative test [16]. Patients affected by aspirin-induced-asthma were also ruled out from the study: the diagnosis was made on basis of positive inhalation (bronchial) provocation test [17]. We finally excluded patients affected by sleep disturbances (patients enrolled showed an AHI score of $<10$ ).

All patients were affected by bilateral NP, had negative skin prick test, and were free from therapy for at least 15 days before the admission to the study. Only nasal lavage with isotonic saline solution was allowed. All subjects performed pulmonary function test, methacholine challenge, bronchial provocation test with aspirin, nasal endoscopy, and nasal cytology. All subjects underwent oral and nasal breath condensate collection one day after previous examinations. Ten patients with NP were investigated 30 days after polypectomy with nasal endoscopy, nasal cytology, at baseline and after decongestion rhinomanometry. They underwent oral and nasal breath condensate collection one day after previous examinations.

\section{Skin test assessment}

Skin prick tests were performed as previously described for common aeroallergens (Lofarma, Italy) [18].

\section{Lung function}

Pulmonary function tests were performed within 1 day of the measurement of the breath condensate being measured. The forced expiratory volume in one second $\left(\mathrm{FEV}_{1}\right)$ and the forced vital capacity (FVC) were measured using a spirometer (Sensormedics, USA). Of three manoeuvers the best value was expressed as a percentage of the predicted normal value.

\section{Nasal endoscopy}

Nasal endoscopy was performed in local anesthesia, using a flexible fiberscope (Vision Science ENT-2000, Massachusetts, USA). We used the modified rhino-fiberscopic score of an International Conference on Nasal Polyposis [14]: $0=$ absence of polyp, $1=$ polyp in medium meato, $2=$ polyp that comes out from meato, $3=$ massive polyposis.

\section{Nasal cytology}

Cytological sampling was performed by scraping with a Rhino-Probe (Apotex Scientific, Inc., Arlington, TX) in the middle part of the lower turbinate, which is located in an area characterised by a significant number of cells, in particular ciliated cells. Fixed with methyl alcohol for 4 minutes, the slides were coloured with the May Grunwald.Giemsa (MGG) staining method.

The microscopic observation with a $400 \mathrm{X}$ enlargement allowed us to check cell distribution, 1000X magnification, in immersion, allowed to distinguish different inflammatory cells. The cellular evaluation was performed through a semi-quantitative study taking in exam 10 microscopic areas.

\section{Basal and after decongestion rhinomanometry}

All individuals underwent a basal and after decongestion rhino-manometry (Rhinomanometer Memphis, amplifon, Italy). The measure unit for the rino-manometry was $\mathrm{PA} / \mathrm{cc} / \mathrm{sec}$ being the pression expressed in Pascal and the flow in cc/sec. The pression flow was established by an Institutional Committee for standardization of rinomanometry at 150 Pascal.

\section{Exhaled breath condensate}

The oral exhaled breath condensate was collected using a condenser which allowed the non-invasive collection of the non-gaseous components from the expired air (EcoScreen, Jaeger, Wurzburg, Germany). Individuals breathed through a mouthpiece and a two-way non-rebreathing valve, which also served as a saliva trap. They were asked to breathe at a normal frequency and tidal volume, wearing a nose clip for a period of 10 minutes. If subjects felt saliva in their mouth they were instructed to swallow it. Nasal exhaled breath condensate was collected applying a facial mask to the EcoScreen, asking subjects to breathe through their nose, with their mouths closed. Condensate, at least $1 \mathrm{ml}$, was collected on ice at $-20^{\circ} \mathrm{C}$, transferred to Eppendorf tubes and stored at $-80^{\circ} \mathrm{C}$ immediately.

\section{Measurement of IL-6 and IL-4}

A specific enzyme immunoassay (EIA) Kit (Cayman Chemical, Ann Arbor, MI) was used to measure IL-6 and IL-4 concentrations in breath condensate. The assays were validated directly by gas chromatography/mass spectrophotometry. The intraassay and interassay variability were $\leq 10 \%$. The detection limits of the assays were $20 \mathrm{pg} / \mathrm{ml}$ and $1.5 \mathrm{pg} / \mathrm{ml}$ respectively. The comparison between oral and nasal concentration of exhaled IL4 and IL-6 were performed according to an assessed method [8]. 
Analysis

Data is expressed as means \pm SD. A MannWhitney test was used to compare groups. Correlations between variables were performed using Spearman's rank correlation test. Significance was defined as a $p$ value of $<0.05$.

\section{Results}

\section{IL-6}

The three subject groups were similar with respect to their lung function $(p<0.01)$. Concentrations of orally and nasally exhaled IL-6 were significantly higher in patients with early nasal polyposis (grade 1) $(4.9 \pm 0.4$ and $4.6 \pm 0.4 \mathrm{pg} / \mathrm{ml})$ and advanced nasal polyposis (grade 2-3) (5.2 \pm 0.2 and $6.6 \pm 0.3)$ compared to healthy controls $(2.8 \pm 0.1$ and 2.7 \pm 0.1 ) (fig. 1). Significantly more elevated concentrations of IL-6 were observed in nasally than in orally exhaled breath condensate of patients with nasal polyposis $(\mathrm{p}<0.005)$, while no differences were found in healthy subjects.

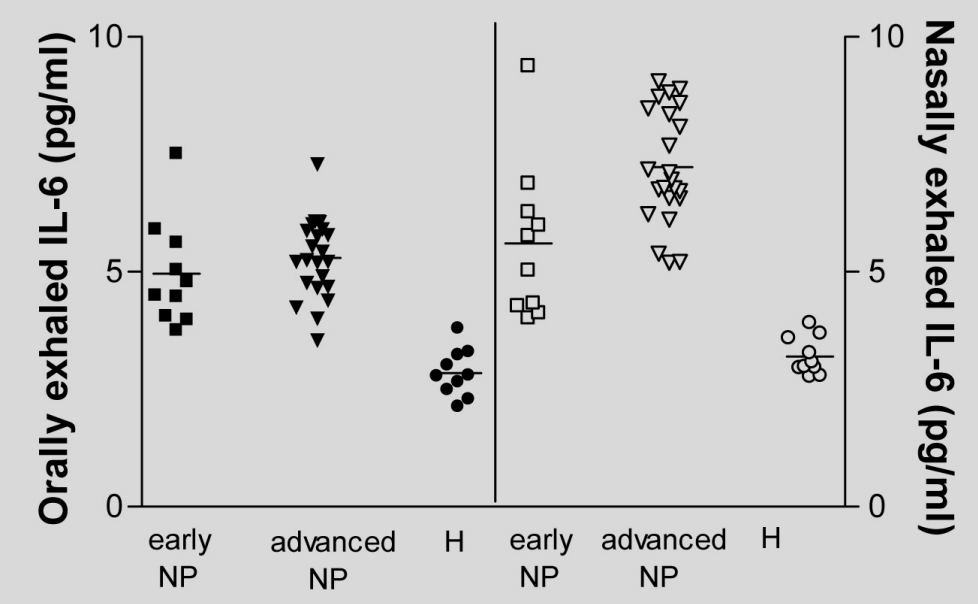

Fig. 1. - IL-6 concentrations in orally and nasally exhaled breath condensate of patients with early nasal polyposis (NP), advanced nasal polyposis (NP) and of healthy controls (H).

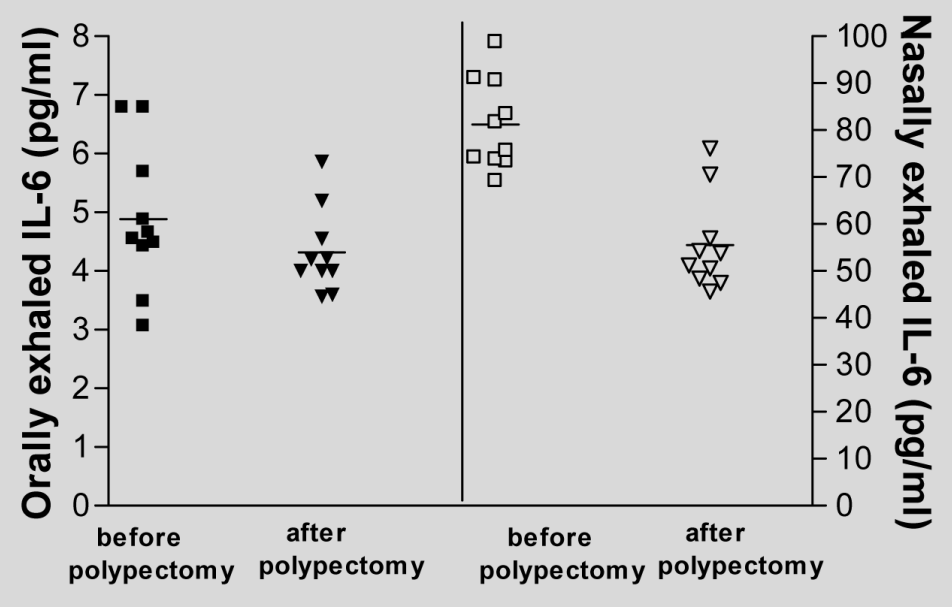

Fig. 2. - IL-6 concentrations in orally and nasally exhaled breath condensate of patients nasal polyposis (NP) before and after polypectomy.
A statistically significant decrease of nasally exhaled IL-6 $(6.5 \pm 1.4$ vs $4.4 \pm 0.2 \mathrm{pg} / \mathrm{ml}, p<0.001)$, but not of orally exhaled IL-6 (4.9 \pm 1.1 vs $4.3 \pm 0.2$ $\mathrm{pg} / \mathrm{ml}$ ) was observed after polypectomy (fig. 2).

A positive correlation between nasally exhaled IL-6 and number of neutrophils in nasal scraping was also observed $(r=0.5, p<0.01)$.

Reproducibility of exhaled IL- 6 measurements was assessed in 10 non-smoking normal (35 \pm 7 years) subjects. In most of measurements, differences between IL- 6 values ranged within \pm 2 SD (mean difference $-0.03 \pm 0.24 \mathrm{pg} / \mathrm{ml}$ ). The co-efficiency of variation for measured IL-6 was $5.9 \%$.

\section{$I L-4$}

Concentrations of orally and nasally exhaled IL-4 were significantly higher in patients with early nasal polyposis $(39.9 \pm 0.8$ and $55.8 \pm 1.9 \mathrm{pg} / \mathrm{ml})$ and in patients with higher grades of polyposis (65.9 \pm 1.7 and $80.9 \pm 2.2$ ), compared to healthy controls (35.8 \pm 1.1 and $37.7 \pm 1.1)$ (fig. 3). Significantly more elevated concentrations of IL-4 were observed in nasally exhaled breath condensate than in orally, either in patients with advanced nasal polyposis $(p<0.0001)$ and in those with early nasal polyposis $(p<0.05)$, while no differences were found in healthy subjects.

The decrease of nasally ex-

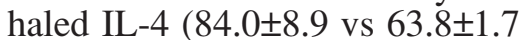
$\mathrm{pg} / \mathrm{ml}, p<0.05$ ) (fig. 4) but not orally exhaled IL-4 (64.6 \pm 8.5 vs $56.1 \pm 3.1 \mathrm{pg} / \mathrm{ml}$ ) after polypectomy was statistically significant.

A positive correlation was observed between nasally exhaled IL-4 and the number of eosinophils in nasal scraping $(\mathrm{r}=0.6, \mathrm{p}<0.005)$.

Reproducibility of exhaled IL-4 measurements was assessed in 10 non-smoking normal $(35 \pm 7$ yr) subjects. In most of measurements, the differences between the two IL-4 values ranged within $\pm 2 \mathrm{SD}$ (mean difference -0.03 $\pm 1.05 \mathrm{pg} / \mathrm{ml}$ ). The co-efficiency of variation for IL-4 measured was $4.2 \%$.

\section{Discussion}

In the present study, we observed significantly higher concentrations of orally and nasally exhaled condensate of IL-6 and IL-4 in patients with early and advanced polyposis compared to healthy controls. However, these concentrations were significantly more elevated in nasally exhaled breath condensate than in orally breath condensate of patients with nasal polyposis. Further- 


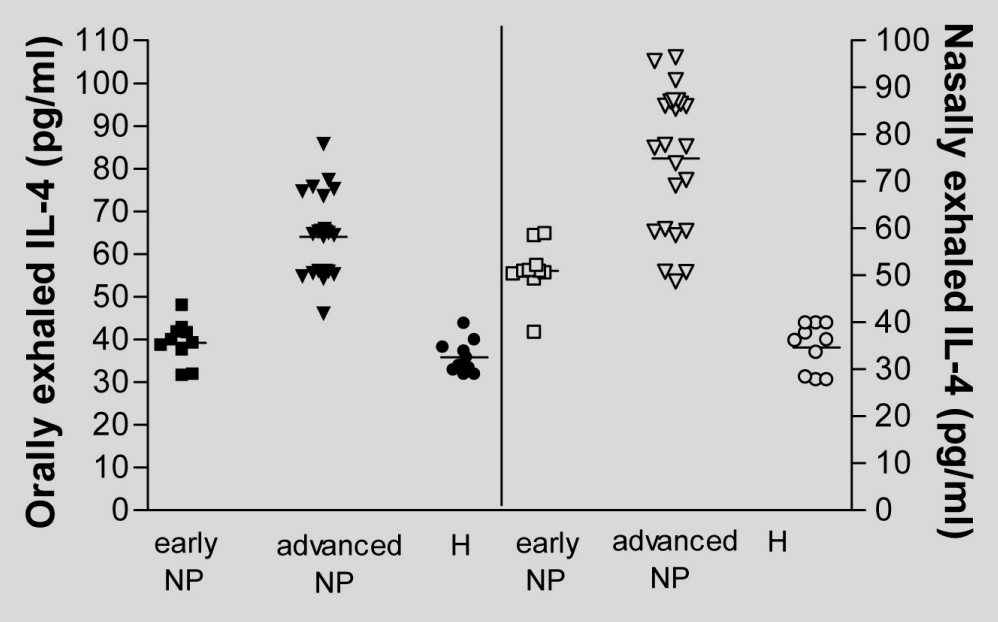

Fig. 3. - IL-4 concentrations in orally and nasally exhaled breath condensate of patients with early nasal polyposis (NP), advanced nasal polyposis (NP) and of healthy controls (H).

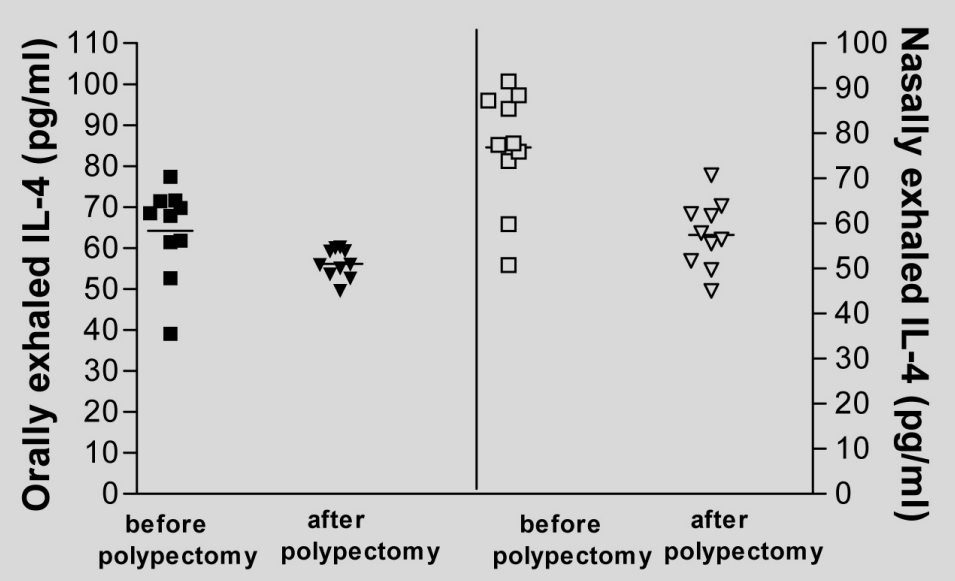

Fig. 4. - IL-4 concentrations in orally and nasally exhaled breath condensate of patients nasal polyposis (NP) before and after polypectomy.

more, we found a significant decrease of nasally exhaled IL-6 and IL-4 after polypectomy, while we did not observe a significant difference in orally exhaled IL-6 and IL-4 after polypectomy. We also demonstrated a positive correlation between nasally exhaled IL-6 with number of neutrophils in nasal scraping and nasally exhaled IL-4 with number of eosinophils in nasal scraping.

Nasal polyposis is characterised by infiltration of inflammatory cells, including mast cells, eosinophils, lymphocytes and neutrophils [19]. Although the exact mechanism elucidating the pathogenesis of NP is not clearly understood, a wide spectrum of cytokines seems to play an important role in developing NP [20]. The pro-inflammatory cytokines IL-4, which modulates the function of eosinophils and is widely produced by $\mathrm{T}$ cells and mast cells, and IL-6, which actively participate to the inflammatory response, and is mainly released by neutrophils and macrophages [21], permanently contribute to perpetuate the mucosal inflammation of patients with NP. IL-4 has been previously utilised to investigate eosinophilic inflammation in nasal lavage of patients with NP [19], is also able to induce cells apoptosis and to promote airway eosinophilia [22]. IL-6 has been widely studied in inflammatory diseases and has been previously found enhanced in nasal polyps epithelial cell-conditioned medium and in polyps tissue of subjects with NP [23]. IL-6 is a multifunctional cytokine: in additional to its modulating role in inflammatory diseases, IL-6 has been suggested to promote collagen deposition and subsequent airway remodelling in NP [24].

To our knowledge, this is the first study in which the exhaled breath condensate assay was used to establish airway inflammation of upper respiratory airways in nasal polyposis. Biochemical analysis of breath condensate is an emerging, non invasive assay to assess airway inflammation on respiratory airways. It is the ultimate way of collecting exhaled breath condensate results in a manner which is easier, safer, does not require active co-operation, and is completely non-invasive [25]. This method has been used largely to study several inflammatory diseases, such as chronic obstructive pulmonary disease (COPD), allergic rhinitis, and asthma [26]. We assessed a modified nasally collection of breath condensate applying a nasal mask to the patients, who were asked to keep their mouth closed during the test. In addition, exhaled air, collected in this way bypassing the mouth, did not present any salivary contamination. In this study, we concomitantly used the oral and the nasal exhaled breath condensate collection to explore the entire upper airway system. The hypothesis that upper airways inflammation can propagate to lower airways in NP has been recently predicted[5]. Lamblin and colleagues described bronchial changes in subjects with NP [6], and subsequent studies confirmed the presence of lower inflammation in NP [27]. Since we found elevated concentrations of IL-4 and IL-6 in oral and in nasal exhaled breath condensate of patients with NP, the upper and lower airways appear, in these condition, to be strongly involved. We further showed that nasally and orally IL-4 and IL-6 levels significantly correlated with eosinophils and neutrophils increase in nasal scrubbing, respectively. Our data confirms the role of these cytokines as markers of eosinophilic and neutrophilic inflammation. In consideration of the strong correlation largely known between eosinophils and exhaled NO and of the usefulness recently proposed of eNO in the clinical and therapeutic follow up and in the control of patients with eosinophilic inflammation the use of eNO in 
association to the exhaled markers analysed could be proposed also in the management of patients with nasal polyposis [28]. Interestingly, the decrease of nasal, but not of oral exhaled condensate concentrations of IL- 6 and IL-4 after polypectomy might correlate with the decline of upper but not lower airway inflammation in these patients. Controversial results exist in the treatment of sinonasal polyps and its involvement in lower airways. In fact, Batra et al demonstrated that the treatment of endoscopic sinus surgery may significantly reduce lower airway inflammation [29], while other authors failed to show this specific relationship [30]. Probably, the effects of polypectomy in reducing lower airway inflammation may require a longer time.

In conclusion, we believe that oral and nasal exhaled condensate of IL- 6 and IL-4 could be applicable as a valid marker of inflammation and oxidative stress in upper and lower airways of patients with nasal polyposis. In addition, being measurable in a simple, non-invasive, economical, and repeatable manner, it might be utilised in clinical practice for identification and monitoring upper and lower airways inflammation in NP, and for the evaluation of post-surgical effects of NP.

\section{References}

1. van der Baan B: Epidemiology and natural history. In Nasal Polyposis: An Inflammatory Disease and Its Treatment. Edited by Mygind N, Lildholt T. Copenhagen, Denmark: Munksgaard; 1997: 13-16.

2. Rudack C, Stoll W, Bachert C. Cytokines in nasal polyposis, acute and chronic sinusitis. Am J Rhinol 1998; 12: $383-8$.

3. Bachert C, Gevaert P, Holtappels G, et al. Total and specific IgE in nasal polyposis is related to local eosinophilic inflammation. J Allergy Clin Immunol 2001; 107: 607-614.

4. Kramer MF, Rasp G. Nasal polyposis: eosinophils and interleukin-5. Allergy 1999; 54: 669-689.

5. Ediger D, Sin BA, Heper A, et al. Airway inflammation in nasal polyposis: immunopathological aspects of relation to asthma. Clin Exp Allergy 2005; 35: 319-26.

6. Lamblin C, Gosset P, Salez F, et al. Eosinophilic airway inflammation in nasal polyposis. J Allergy Clin Immunol 1999; 104: 85-92.

7. Scheiderler L, Manke HG, Schwulera U, et al. Detection of nonvolatile macromolecules in breath. A possible diagnostic tool? Am Rev Respir Dis 1993; 148: 778-84.

8. Griese M, Latzin P, Beck J. A non-invasive method to collect nasally exhaled air condensate in humans of all ages. Eur J Clin Invest 2001; 31: 915-20.

9. Cunningham S, McColm JR, Ho LP, et al. Measurement of inflammatory markers in the breath condensate of children with cystic fibrosis. Eur Respir J 2000; 15 : 955-7.

10. Garey KW, Neuhauser MM, Robbins RA, et al. Markers of inflammation in exhaled breath condensate of young healthy smokers. Chest 2004; 125: 22-6.

11. Carpagnano GE, Kharitonov SA, Resta O, et al. Increased 8-isoprostane and interleukin-6 in breath condensate of obstructive sleep apnea patients. Chest 2002; 122: 1162-7.

12. McRae K, De Perrot M, Fischer S, et al. Detection of IL-10 in the exhaled breath condensate, plasma and tis- sue during ischemia-reperfusion injury in experimental lung transplantation. J Heart Lung Transplant 2001; 20: 184 .

13. Carpagnano GE, Barnes PJ, Geddes DM, et al. Increased leukotriene B4 and interleukin-6 in exhaled breath condensate in cystic fibrosis. Am J Respir Crit Care Med 2003; 167: 1109-12.

14. Passali D, Bellussi L, Hassan HA, et al. Consensus Conference on Nasal Polyposis. Acta Otorhinolaryngol Ital 2004 Apr; 24 (2 Suppl 77): 3-61.

15. Makino S, Adachi M, Ago Y, et al and Research Group for Asthma Prevention and Management Guidelines. Definition, diagnosis, disease types, and classification of asthma. Int Arch Allergy Immunol 2005; 136 Suppl 1: 3-4.

16. Chinn S, Schouten JP. Reproducibility of non-specific bronchial challenge in adults: implications for design, analysis and interpretation of clinical and epidemiological studies. Thorax 2005; 60: 395-400.

17. Melillo G, Balzano G, Bianco S, et al. Report of the INTERASMA Working Group on Standardization of Inhalation Provocation Tests in Aspirin-induced Asthma. Oral and inhalation provocation tests for the diagnosis of aspirin-induced asthma. Allergy 2001; 56: 899-911.

18. Deborg S., Frew A. Position paper: Allergen standardization and skin tests. Allergy 1993; 48 (suppl 14): 49-82.

19. Di Lorenzo G, Drago A, Esposito, et al. Measurement of inflammatory mediators of mast cells and eosinophils in native nasal lavage fluid in nasal polyposis. Int Arch Allergy Immunol 2001; 125: 164-75.

20. Singh H, Ballow M. Role of cytokines in nasal polyposis. J Investig Allergol Clin Immunol 2003; 13: 6-11.

21. Bachert C, Gevaert P, Holtappels G, et al. Mediators in nasal polyposis. Curr Allergy Asthma Rep 2002; 2: 481-7.

22. Cohn L, Herrick C, Niu N, et al. IL-4 promotes airway eosinophilia by suppressing IFN-gamma production: defining a novel role for IFN-gamma in the regulation of allergic airway inflammation. J Immunol 2001; 166: 2760-7.

23. Mullol J, Xaubet A, Gaya A, et al. Cytokine gene expression and release from epithelial cells. A comparison study between healthy nasal mucosa and nasal polyps. Clin Exp Allergy 1995; 25: 607-15.

24. Liu CM, Hong CY, Shun CT, et al. Inducible cyclooxygenase and interleukin 6 gene expressions in nasal polyp fibroblasts: possible implication in the pathogenesis of nasal polyposis. Arch Otolaryngol Head Neck Surg 2002; 128: 945-51.

25. Horvath I, Hunt J, Barnes PJ. Exhaled breath condensate: methodological recommendations and unresolved questions. Eur Respir J 2005; 26: 523-48.

26. Mutlu GM, Garey KW, Robbins RA, et al Collection and analysis of exhaled breath condensate in humans. Am J Respir Crit Care Med 2001; 164: 731-7.

27. Borish L. The role of leukotrienes in upper and lower airway inflammation and the implications for treatment. Ann Allergy Asthma Immunol 2002; 88 (4 Suppl 1): 16-22.

28. Malerba M, Ragnoli B, Radaeli A, Tntucci C. Usefulness of exhaled nitric oxide and sputum eosinophils in the long term control of eosinophilic asthma. Chest 2008; 134: 733-9.

29. Batra PS, Kern RC, Tripathi A, Conley DB, et al. Outcome analysis of endoscopic sinus surgery in patients with nasal polyps and asthma. Laryngoscope 2003; 113: 1703-6.

30. Dufour X, Bedier A, Ferrie JC, et al. Diffuse nasal polyposis and endonasal endoscopic surgery: long-term results, a 65-case study. Laryngoscope 2004; 114: 1982-7. 